\title{
Posttraumatische Belastungssymptomatik und Gedankenkontrollstrategien bei Verkehrsunfallopfern
}

\section{Posttraumatic Stress Symptomatology and Thought Control Strategies in Victims of Motor Vehicle Accidents}

\author{
$\begin{array}{lllll}\text { A. Poldrack } & \text { A. Maercker } & \text { J. Margraf } & \text { D. Kloten } & \text { J. M. Gavlik } \\ \text { c } & \text { H. Zwipp } & \end{array}$ \\ ${ }^{a}$ Christoph-Dornier-Stiftung für Klinische Psychologie, Institut Dresden, ${ }^{b}$ Klinische Psychologie und Psychotherapie und ${ }^{\mathrm{c} K l i n i k}$ für Unfall- \\ und Wiederherstellungschirurgie des Universitätsklinikums der Technischen Universität Dresden
}

\section{Schlüsselwörter}

Posttraumatische Belastungsstörung .

Verkehrsunfallopfer · Psychotraumatologie

\section{Zusammenfassung}

In einer längsschnittlichen Untersuchung wurden $64 \mathrm{~Pa}$ tienten der unfallchirurgischen Abteilung einer Universitätsklinik 10 Tage und 3 Monate nach einem Verkehrsunfall untersucht. Erhoben wurden die posttraumatische Belastungssymptomatik und Gedankenkontrollstrategien. Zum ersten Untersuchungszeitpunkt (10 Tage) zeigt sich eine der Posttraumatischen Belastungsstörung (PTB) äquivalente Belastungssymptomatik bei $14,1 \%$ und nach 3 Monaten eine PTB-Prävalenz von 39,1\%. Es zeigen sich Zusammenhänge zwischen einigen Gedankenkontrollstrategien und PTB-Symptomatik nach 10 Tagen und, in schwächerer Ausprägung auch nach 3 Monaten. Im Ergebnis einer explorativen Auswertung zeigt sich, daß insbesondere die Strategien "Ablenkung" und "Sorgen" eine relevante Rolle im Zusammenhang mit der PTB-Symptomatik spielen.

\section{Hintergrund}

Verkehrsunfälle gehören zu den häufigsten Ursachen von Tod und Verletzung. In einer Untersuchung von Norris [1992] fand sich, daß 23,4\% der Allgemeinbevölkerung in ihrem Leben einen Verkehrsunfall erlebt hatten. Dies deckt sich mit Ergebnissen der größten vorliegenden epidemiologischen Studie, in der $19 \%$ der Teilnehmer angaben, schon einmal einen Verkehrsunfall erlebt zu haben [Kessler et al., 1995]. Im Jahre 1995 wurden in Deutschland 2,23 Mio. Straßenverkehrsunfälle polizeilich erfaßt. Darunter waren 388.000 Un-

\section{Key Words}

Posttraumatic stress disorder - Motor vehicle accident victims - Psychotraumatology

\section{Abstract}

In a longitudinal survey 64 inpatients of an emergency clinic were examined 1 week as well as 3 months after a motor vehicle accident. Posttraumatic stress symptoms and strategies of thought control were assessed. Ten days after the accident, a posttraumatic stress symptomatology is observable in $14.1 \%$ of patients, 3 months after the accident the prevalence of posttraumatic stress disorder (PTSD) is $39.1 \%$.

Strategies of thought control are correlated with posttraumatic symptomatology 10 days after the accident and after 3 months. The result of an explorative data analysis shows an important role of the control strategies 'distraction' and 'worry' in correlation with posttraumatic stress symptoms.

\begin{tabular}{ll}
\hline KARGER & (c) 1999 S. Karger GmbH, Freiburg \\
Fax +497614520714 & Accessible online at: \\
$\begin{array}{l}\text { E-mail Information@Karger.de } \\
\text { www.karger.com }\end{array}$ & www.karger.com/journals/ver
\end{tabular}

fälle mit Personenschaden, bei denen 512.141 Menschen verletzt wurden (Statistisches Bundesamt, 1998). Man kann also davon ausgehen, daß Verkehrsunfälle zu den relevantesten Trauma-auslösenden Ereignissen in der westlichen Industriegesellschaft zählen, wenngleich die bisherigen psychotraumatologischen Forschungsergebnisse zeigen, daß die Wahrscheinlichkeit, nach einem Unfall eine Posttraumatische Belastungsstörung (PTB) auszubilden, bedeutend geringer ist als nach anderen traumatischen Ereignissen (z.B. Vergewaltigungen, Geiselnahme, vgl. Maercker, 1997).

Es liegen bisher mehrere Querschnittsuntersuchungen zu Er-

\footnotetext{
Dipl.Psych. A. Poldrack

Psychotherapeutische Praxisgemeinschaft

Conradstraße 4

D-01097 Dresden
}

Tel. 0351-8011548 
scheinungsbild und Prävalenz der PTB bei Verkehrsunfallopfern vor, deren Ergebnisse allerdings ein heterogenes Bild zeichnen. Dagegen existiert abgesehen vom retrospektiven Studien nur eine überschaubare Anzahl von Längsschnittstudien, die den Verlauf oder die Prädiktion von PTB untersuchten [z.B. Blanchard et al., 1996; Ehlers et al., 1998; Koren et al., 1999; Mayou et al., 1993].

\section{Posttraumatische Belastungsstörung nach Verkehrsunfällen}

Die Studien zur Prävalenz von PTB nach Verkehrsunfällen weisen uneinheitliche Ergebnisse auf. Die gefundenen Prävalenzraten reichen von 1\% [Malt, 1988] bis hin zu 49\% [Hickling und Blanchard, 1992]. Gründe für die Heterogenität der Ergebnisse sind unterschiedliche Nachuntersuchungszeiträume, unscharfe Kriterien bei der Erfassung relevanter Variablen, wie z.B. der PTB-Diagnose oder der Verletzungsschwere, selektive Stichprobenrekrutierung und zum Teil retrospektives Untersuchungsdesign.

In einer in Deutschland durchgeführten Längsschnittstudie fand sich nach 6 Monaten eine Prävalenzrate von 8,2\% für das Vollbild einer PTB und 10,2\% für eine subsyndromale PTB in der Definition von Blanchard [Frommberger et al., 1998; Nyberg et al., 1998].

\section{Traumaschwere als traditioneller Prädiktor}

Traditionell wurde die Trauma- bzw. Unfallschwere als Risikofaktor für das Entwickeln einer PTB angesehen [March, 1993]. Studien an Vergewaltigungsopfern zeigten, daß mit der Traumaschwere auch die PTB-Häufigkeit ansteigt. Als relevantes Kriterium für die Traumaschwere fand man unter anderem das Ausmaß körperlicher Verletzungen [Kilpatrick et al., 1989]. Es scheint demnach bei Vergewaltigungsopfern eine Dosis-Wirkungs-Beziehung zu geben, wonach besonders schwere Traumatisierungen besonders ausgeprägte Belastungssymptome nach sich ziehen.

Die Befundlage zur Dosis-Wirkungs-Beziehung bei Verkehrsunfällen ist dagegen uneinheitlich. Dies liegt nicht zuletzt auch daran, daß es bisher nicht gelungen ist, ein objektives $\mathrm{Maß}$ für die Unfallschwere zu entwickeln. In vielen Studien werden zwar Unfallmerkmale miterhoben, es ist aber unklar, welche Unfallmerkmale relevant sind. In einigen Studien wurde deshalb das Ausmaß der körperlichen Verletzungen als ein Aspekt der Traumaschwere herangezogen. Die Ergebnisse einiger dieser Studien [z.B. Blanchard et al., 1995; Malt et al., 1993] lassen den Schluß zu, daß es einen positiven Zusammenhang gibt zwischen der Verletzungsschwere und der Wahrscheinlichkeit, eine PTB zu entwickeln. Die Mehrzahl der Studien zu diesem Thema [z.B. Bryant et al., 1995; Ehlers et al., 1998; Feinsten, 1991; Mayou et al., 1993] können diesen Zusammenhang jedoch nicht bestätigen, wobei zu berücksichtigen ist, daß in einigen Studien die Erfassung der Verletzungsschwere methodische Mängel aufwies. Mittlerweile gibt es empirische Hinweise, daß die initiale Reaktion bzw. initiale kognitive Veränderungen einen größeren Effekt zu haben scheinen als der klassische «Dosis-Wirkungs-Zusammenhang» [Maercker, 1998].

\section{Gedankenkontrolle als Aufrechterhaltungsfaktor für PTB}

Das Auftreten unerwünschter intrusiver Gedanken ist bis zu einem gewissen Grade normal und weit verbreitet. Problematisch für die Betroffenen wird dies meist erst dann, wenn diese Gedanken subjektiv als schwer kontrollierbar erlebt werden und im eigentlichen Sinne als Intrusionen bezeichnet werden können [Steil et al., 1997]. Solche Intrusionen sind ein zentrales Merkmal der PTB. Daneben finden sich diese klinischen Phänomene auch bei Angststörungen wie der Generalisierten Angststörung oder bei Zwangsstörungen. Das Auftreten unerwünschter Gedanken zieht den Wunsch, diese zu kontrollieren, geradezu zwangsläufig nach sich. Dieser Ansatz hat nicht zuletzt auch in psychotherapeutischen Interventionstechniken wie Gedankenstopp, kognitiver Umbenennung und kontrollierten Grübelphasen seinen Niederschlag gefunden.

Seit einiger Zeit sind die Prozesse des Umgangs mit unerwünschten Gedanken Gegenstand klinisch-psychologischer Forschungsbemühungen. Zunächst richtete sich die Aufmerksamkeit auf die Strategie der Gedankenunterdrückung. Gedankenunterdrückung wird dabei als Versuch definiert, nicht an den betreffenden Gedanken zu denken [Wegner et al., 1987]. Eine Reihe von Studien, die dazu durchgeführt wurden, kommen zu dem Schluß, daß Gedankenunterdrückung einen paradoxen Effekt hat, d.h. daß der unterdrückte Gedanke vermehrt auftaucht [z.B. Clark und Purdon, 1993; Salkovskis und Campbell, 1994]. Eher unklar war lange Zeit, mit welchen «Techniken» Menschen intrusive Gedanken unterdrücken. Wells und Davies [1994] fanden in ihrer Studie an 229 Studenten fünf unabhängige Gedankenkontrollstrategien: Ablenkung, soziale Kontrolle, Sorgen, Selbstbestrafung und Umbenennung, die auch in der vorliegenden Studie untersucht werden.

\section{Untersuchungsziele}

Ausgehend von der Betrachtung der bisherigen Ergebnisse war das Ziel der vorliegenden Studie, in einem naturalistischen, d.h., dem klinischen Alltag nahen Setting im Rahmen einer Längsschnittstudie die Auftretenshäufigkeit posttraumatischer Belastungssymptomatik zu untersuchen sowie das Erscheinungsbild aufzuzeigen. Darüber hinaus soll der Beitrag der körperlichen Verletzungsschwere zur Entwicklung einer PTB eingeschätzt werden, wobei angenommen wird, daß dieser relativ gering ist. Im Mittelpunkt des Interesses steht die Untersuchung des Zusammenhanges von Gedankenkontrollstrategien und PTB-Symptomatik. Welche Gedankenkontrollstrategien dabei eine besondere Rolle spielen, bleibt offen, so daß die Untersuchung der einzelnen Gedankenkontrollstrategien explorativ bleibt. 
Aus früheren Studien liegen Hinweise vor, daß Intrusionen, die kurz nach dem Unfall berichtet werden, zur Aufrechterhaltung posttraumatischer Belastungssymptomatik beitragen können. Andererseits wird den Strategien, mit denen Verkehrsunfallopfer die wiederkehrenden Erinnerungen zu kontrollieren versuchen, zentrale Bedeutung beigemessen. Diese Zusammenhänge sollen in dieser Studie einer näheren Betrachtung unterzogen werden.

\section{Methode}

\section{Stichprobe}

Die untersuchte Stichprobe umfaßt 75 Patienten, die innerhalb 1 Woche nach dem Unfall untersucht wurden. Ausgeschlossen von der Untersuchung waren a) Unfallopfer, die wegen ZNS- bzw. Schädelverletzungen auf die Intensivtherapiestation eingeliefert worden waren, b) Personen, die in suizidaler Absicht einen Verkehrsunfall verursacht hatten, sowie c) Probanden, bei denen keine ausreichenden Kenntnisse der deutschen Sprache vorlagen. Von den zum ersten Zeitpunkt untersuchten 75 Patienten konnten 64 Personen für die Nachuntersuchung nach 3 Monaten gewonnen werden. Die 11 fehlenden Probanden verweigerten entweder die erneute Mitarbeit oder waren nicht mehr erreichbar. Die folgenden Ergebnisse basieren auf den Daten der 64 Patienten.

Alle Patienten waren zum Zeitpunkt der ersten Untersuchung stationäre Patienten der unfallchirurgischen Abteilung des Universitätsklinikums der Technischen Universität Dresden, einer großen Unfallklinik mit Normalversorgungsprofil. Tabelle 1 zeigt die Stichprobencharakteristika. Das Durchschnittsalter der untersuchten Stichprobe lag bei 31,7 Jahren (Median 27,0; s = 14,9; Bereich: 15,0-73,0). Die Auflistung der Unfallbeteiligungsarten zeigt, daß der überwiegende Teil der Patienten als Fahrzeugführer am Unfall beteiligt war.

\section{Untersuchungsablauf}

Die Patienten wurden innerhalb der ersten Woche nach ihrem Unfall auf der unfallchirurgischen Station kontaktiert. Sie wurden über das Ziel der Studie aufgeklärt und um Mitarbeit gebeten. Die nachfolgend beschriebenen Fragebögen (PDS, IES-R) sowie ein soziodemographischer Fragebogen und ein Fragebogen zum Unfallhergang) wurden von den Patienten selbst ausgefüllt. 3 Monate nach dem Unfall setzten wir uns schriftlich mit den Patienten in Verbindung und sandten ihnen dieselben Fragebögen noch einmal zu. Es fanden bis zu drei Nachfaßversuche statt, bei denen die Patienten an die Rücksendung des Fragebogens erinnert wurden. Die Rücklaufquote betrug zum ersten Untersuchungszeitpunkt $76,5 \%$ und zum zweiten $85,3 \%$.

\section{Erhebung der PTB-Symptomatik}

Die von Foa [1995] entwickelte und validierte und von Ehlers et al. [1996] übersetzte «Posttraumatic Diagnostic Scale» (PDS) ist in der Lage, sowohl die Art des Ereignisses zu spezifizieren als auch die, aus den Symptomen resultierenden, Beeinträchtigungen innerhalb verschiedener Lebensbereiche zu erfassen. Dies geschieht anhand der Diagnosekriterien des DSM-IV [American Psychiatric Association, 1994].

Die PDS besteht aus insgesamt drei Teilen: (1) Eine Checkliste, in der gefragt wird, wie oft der Patient welche traumatischen Ereignisse entweder selbst oder als Zeuge erlebt hat. Der Patient soll angeben, welches der erlebten Ereignisse das für ihn subjektiv schlimmste war. (2) Im zweiten Teil werden anhand von 17 Items die mit dem DSM-IV übereinstimmenden PTB-Symptome erfaßt. Die Häufigkeit jedes Symptoms im vergangenen Monat wird auf einer 4stufigen Skala geratet $(0=$ überhaupt nicht oder nur einmal im letzten Monat; $1=$ einmal pro Woche oder
Tab. 1. Stichprobencharakteristik $(\mathrm{N}=64)$

\begin{tabular}{lr}
\hline Merkmale & $\%$ \\
\hline Unfallbeteiligung & \\
Fahrzeugführer & 58,2 \\
Beifahrer/Sozius & 14,5 \\
Fußgänger & 7,3 \\
Radfahrer & 20,0 \\
& \\
Geschlecht & \\
weiblich & 32,8 \\
männlich & 67,8 \\
& \\
Erwerbstätigkeit & \\
Vollzeit & 50,5 \\
Teilzeit & 7,8 \\
Schüler/Studenten & 21,9 \\
Arbeitslos & 6,8 \\
Vorruhestand/Rente & 13,0 \\
\hline &
\end{tabular}

seltener/manchmal; $2=2$ bis 4 mal pro Woche/die Hälfte der Zeit; $3=5$ mal oder öfter pro Woche/fast immer). Über diese 17 Items kann ein Summenscore gebildet werden, um die Schwere der Symptomatik abzubilden. (3) Im dritten und letzten Teil werden die Auswirkungen der Symptomatik auf verschiedene Lebensbereiche in Form von Beeinträchtigungsratings erfragt.

IES-R (Impact of Event Scale - revised, dt. Version von Maercker und Schützwohl, 1998):

Der von Weiss und Marmar [1997] entwickelte IES-R ist ein international weit verbreitetes Meßinstrument. Er besteht aus 22 Items, die sich drei psychometrisch konstruierten Subskalen zuordnen lassen: Intrusion, Vermeidung und Hyperarousal. Die Antworten auf der 4stufigen Skala werden verrechnet und ergeben für die Subskalen «Intrusionen» sowie «Hyperarousal» einen Wertebereich von 0 bis 35 , für die Subskala «Vermeidung» einen Wertebereich von 0 bis 40 . Der IES-R erlaubt keine diagnostische Zuordnung auf direktem Wege, da die 22 Items des IES-R nicht vollständig mit den 17 DSM-Symptomen übereinstimmen und lediglich Häufigkeiten erfaßt werden. Eine Regressionsformel ermöglicht jedoch eine Abschätzung der Diagnosezuordnung. Maercker und Schützwohl [1998] berichten über zufriedenstellende bis gute teststatistische Kennwerte für die deutsche Version.

\section{Erhebung der Verletzungsschwere}

ISS (Injury Severity Score) [AMA Commitee on Medical Aspects of Automotive Safety, 1971; Baker et al., 1974]: Der ISS wird zur Abschätzung des Schweregrades des Unfalls herangezogen und ist ein Fremdrating, bei dem ein Arzt anhand klar operationalisierter Kriterien die Schwere der Verletzungen in fünf definierten Körper- bzw. Organregionen vornimmt. Die Quadratsumme aus den Werten der drei am schwersten betroffenen Regionen bildet dann den ISS-Wert, der von der American Medical Association als Standard entwickelt wurde und international in der Unfallmedizin recht weit verbreitet ist. In der vorliegenden Studie kann der ISS Werte zwischen 0 und 75 annehmen, wobei Werte über 40 eher selten auf einer unfallchirurgischen Station zu finden sind. Diese sehr schwer verletzten Patienten werden in der Regel auf einer Intensivtherapiestation betreut.

\section{Erhebung der Gedankenkontrolle}

TCQ (Thought Control Questionnaire) [Wells und Davies, 1994; deutsche Übersetzung: Fehm, 1997]: Dieser 30-Item-Fragebogen wurde entwickelt, um die Häufigkeit des Einsatzes von fünf Gedankenkontrollstra- 
tegien zu erheben: Ablenkung (z.B. an etwas Angenehmes denken), soziale Kontrolle (z.B. Kommunikation mit anderen über die intrusiven Gedanken), Sorgen (z.B. Grübeln über intrusive Gedanken), Selbstbestrafung (z.B. Ärger über sich selbst) und Neubewertung (z.B. konstruktive Umbewertung). Jede Subskala besteht aus sechs Items, die auf einer 4stufigen Skala eingeschätzt werden können $(1=$ nie, $4=$ meistens/immer $)$. Die interne Konsistenz der Originalversion liegt zwischen 0,64 und 0,83. Fehm [1997] konnte die Fünf-Faktoren-Lösung von Wells und Davies [1994] weitgehend replizieren. Auch die geringen Interkorrelationen zwischen den Subskalen ließen sich bestätigen. Als befriedigend wird die Retest-Reliabilität der deutschen Version eingeschätzt. Sie liegt zum Teil deutlich unter den Werten für die Originalversion $(0,67$ bis 0,83 für ein 6wöchiges Retest-Intervall). Die Validität der Skala wird von Fehm [1997] ausreichend, insgesamt jedoch nicht unproblematisch eingeschätzt. Es werden moderate signifikante Korrelationen der Subskalen «Sorgen» ( $\mathrm{r}=0,16-0,32)$ und «Bestrafung» $(\mathrm{r}=0,20-0,41)$ zu mehreren Subskalen des SCL-90-R berichtet. Hier wäre eine bessere divergente Validität wünschenswert. Insgesamt wird der TCQ als ein Meßinstrument mit ausreichenden Gütekriterien betrachtet, das wegen seiner guten Handhabbarkeit vor allem die Feldforschung geeignet ist.

\section{Statistische Analyse}

Eine PTB-Diagnose wurde dann vergeben, wenn ein Patient 3 Monate nach dem Unfall im PDS alle DSM-IV-Kriterien (A bis F) für eine PTB erfüllte. Traf dies bereits 10 Tage nach dem Unfall zu (mit Ausnahme des Zeitkriteriums E), wurde dies als «PTB-Äquivalent» eingestuft. Eine Vergabe der Diagnose Akute Belastungsstörung ist anhand des PDS nicht möglich, da einige für diese Störung typische Symptome (z.B. Dissoziation) nicht ausreichend erfaßt werden.

Die Zusammenhänge zwischen der Verletzungsschwere und der posttraumatischen Belastungssymptomatik wurden als Rangkorrelationskoeffizienten berechnet. Parallel wurde, um einen eventuell vorliegenden nichtlinearen Zusammenhang ebenfalls zu überprüfen, ein t-Test für unabhängige Stichproben durchgeführt, bei dem die mittlere Verletzungsschwere, erhoben zum ersten Meßzeitpunkt, in den beiden Diagnosekategorien «kein $\mathrm{PTB}$ » und «PTB», erhoben zum zweiten Meßzeitpunkt, verglichen wurde.

Korrelationsanalysen wurden durchgeführt zur Überprüfung des Zusammenhangs von Gedankenkontrollstrategien und posttraumatischer Symptomatik. Abschließend wurde eine Regressionsanalyse durchgeführt, um den prädiktiven Wert einzelner Gedankenkontrollstrategien zu untersuchen. Es kam eine multiple lineare Regression nach der Einschlußmethode zum Einsatz.

\section{Ergebnisse}

\section{Prävalenz posttraumatischer Belastungssymptomatik}

\section{DSM-IV-Diagnose}

Nahezu alle untersuchten Patienten erlebten bzw. beobachteten drohende oder tatsächliche Verletzung oder Tod. Dreiviertel der Probanden reagierten mit intensiver Furcht, Hilflosigkeit oder Entsetzen auf den Verkehrsunfall. Der überwiegende Teil der untersuchten Verkehrsunfallopfer berichtet darüber hinaus über posttraumatische Belastungssymptome. Wie in Tabelle 2 aufgeführt, unterscheiden sich die Auftretenshäufigkeiten der einzelnen Symptomkategorien deutlich: Mit Abstand am häufigsten erfüllten Patienten das DSM-IV-Symptomcluster «Intrusionen» $(68,8 \%$ nach 1 Woche, $84,4 \%$ nach 3 Monaten).
Tab. 2. Diagnosekriterien und Anteil der Patienten in Prozent $(\mathrm{N}=64)$

\begin{tabular}{lll}
\hline Kriterium & 1 Woche & 3 Monate \\
\hline A1 (Bedrohung) & 96,6 & 96,6 \\
A2 (Reaktion) & 75,4 & 75,4 \\
B (Intrusionen) & 68,8 & 84,4 \\
C (Vermeidung) & 51,6 & 48,4 \\
D (Hyperarousal) & 37,5 & 54,7 \\
B-D gesamt & 29,7 & 39,1 \\
F (Beeinträchtigung) & 57,8 & 67,2 \\
DSM-IV-Diagnose & $(14,1)^{\mathrm{a}}$ & 39,1 \\
\hline
\end{tabular}

aentsprechend DSM-IV ohne Zeitkriterium.

39,1\% der Patienten erfüllten nach 3 Monaten die Kriterien der PTB-Diagnose. Nach 10 Tagen hatten lediglich 14,1\% ein PTB-Äquivalent. Zwischen der Kontrollvariablen Geschlecht und den drei PTB-Symptomclustern lassen sich lediglich zum ersten Untersuchungszeitpunkt moderate signifikante Korrelationen finden. Nach 3 Monaten korreliert lediglich das Vorhandensein einer PTB, aber nicht mehr die einzelnen Symptomcluster mit dem Geschlecht. Dabei sind Frauen tendenziell häufiger von PTB-Symptomatik betroffen. Das Alter korreliert lediglich mit dem Vorhandensein einer PTB nach 3 Monaten (Tab. 3).

\section{Verlauf}

Betrachtet man die Veränderungen der PTB-Diagnosezuordnung zwischen den beiden Untersuchungszeitpunkten, so zeigt sich folgendes: Ausgehend von einem gesunden Status am ersten Zeitpunkt entwickelten 35,9\% ( $\mathrm{N}=23)$ eine PTB zum zweiten Zeitpunkt, bei 53,1\% ( $\mathrm{N}=34)$ blieb der Symptomstatus unverändert und bei $10,9 \%(\mathrm{~N}=7)$ remittierte die zum ersten Untersuchungszeitpunkt vorliegende PTB-äquivalente Störung.

Zwei t-Tests für abhängige Stichproben erbrachten sowohl für die PTB-Gruppe als auch für Nicht-PTB-Gruppe signifikante Unterschiede zwischen dem mittleren PDS-Wert $\mathrm{zu} \mathrm{t}_{1}$ und $\mathrm{zu}$ $\mathrm{t}_{2}$. Bei der PTB-Gruppe lag der durchschnittliche PDS-Wert $\mathrm{zu}_{2}$ signifikant höher als zu $\mathrm{t}_{1}(\mathrm{t}=-2,89 ; \mathrm{df}=24 ; \mathrm{p}=0,008)$, bei der Nicht-PTB-Gruppe lag er signifikant unter dem Wert zum ersten Zeitpunkt $(\mathrm{t}=3,36 ; \mathrm{df}=38 ; \mathrm{p}=0,002)$.

\section{Verletzungsschwere}

In der Stichprobe überwiegen die Patienten mit mittelgradigen Verletzungen (ISS 1-12, 48,7\%). Schwere (ISS 13-19) und sehr schwere Verletzungen (ISS $>20$ ) treten etwa gleich häufig auf (27,6 bzw. 22,4\%). Der Mittelwert des ISS liegt bei 13,85 (s = 9,03; Min. 1,0; Max. 43,0). 
Tab. 3. Korrelationen zwischen Prädiktor-Variablen und IES-R-Skalen bzw. PTB-Diagnose nach DSM-IV $(\mathrm{N}=64)$

\begin{tabular}{|c|c|c|c|c|c|c|c|}
\hline & \multicolumn{3}{|l|}{1 Woche } & \multicolumn{4}{|l|}{3 Monate } \\
\hline & Intrusionen & Vermeidung & Hyperarousal & Intrusionen & Vermeidung & Hyperarousal & Diagnose \\
\hline Alter & 0,12 & 0,29 & 0,08 & 0,19 & 0,11 & 0,05 & $0,28 *$ \\
\hline Geschlecht & $0,39 * *$ & $0,32 *$ & $0,23^{+}$ & 0,10 & 0,19 & 0,14 & $0,25 *$ \\
\hline Verletzunggsschwere & 0,12 & 0,14 & 0,07 & $-0,17$ & $-0,02$ & $-0,07$ & $-0,01$ \\
\hline Neubewertung (TCQ) & $0,25^{*}$ & $0,30 *$ & $0,26^{*}$ & $-0,03$ & 0,07 & 0,07 & 0,15 \\
\hline Sorgen (TCQ) & $0,40 * *$ & $0,52 * * *$ & $0,47 * * *$ & $-0,27 *$ & $-0,27 *$ & $-0,18$ & $-0,05$ \\
\hline Bestrafung (TCQ) & $0,33 * *$ & $0,40 * *$ & $0,37 * *$ & $-0,14$ & $-0,15$ & $-0,22^{+}$ & $-0,12$ \\
\hline Soziale Kontrolle (TCQ) & $0,23^{+}$ & $0,42 * *$ & $0,32 *$ & $-0,04$ & 0,04 & $-0,01$ & 0,12 \\
\hline
\end{tabular}

${ }^{+} \mathrm{p}<0,08, * \mathrm{p}<0,05, * * \mathrm{p}<0,01, * * * \mathrm{p}<0,001$.

Die Verletzungsschwere korreliert zum ersten Untersuchungszeitpunkt kurz nach dem Unfall nicht signifikant mit spezifischer Belastungssymptomatik (Tab. 3), was sich in ähnlicher Form zum zweiten Untersuchungszeitpunkt nach 3 Monaten findet.

Um auch eventuelle nichtlineare Zusammenhänge zu prüfen, wurde die Einteilung der Verletzungsschwere nach drei Schweregraden auf Unterschiede im Ausmaß der PTB-Symptomatik varianzanalytisch untersucht. Es fanden sich dabei weder zum ersten noch zum zweiten Untersuchungszeitpunkt signifikante Unterschiede zwischen den drei Gruppen hinsichtlich des PDS-Symptomsummenscores $\left(\mathrm{t}_{1}: \mathrm{F}=1,05 ; \mathrm{df}=2\right.$; $\left.\mathrm{p}=0,35 ; \mathrm{t}_{2}: \mathrm{F}=1,26 ; \mathrm{df}=2 ; \mathrm{p}=0,29\right)$ und keiner der insgesamt 6 PDS- bzw. IES-R-Subskalen.

\section{Gedankenkontrolle}

Die erhobenen Gedankenkontrollstrategien korrelieren zum ersten Untersuchungszeitpunkt zum Teil hochsignifikant und in erheblicher Höhe mit den Symptomclustern. So stehen die Strategien «Ablenkung» und «Neubewertung» sowohl mit dem Auftreten von Intrusionen, Vermeidung als auch mit Hyperarousal in einem positiven Zusammenhang. Die Strategie «Sorgen» korreliert mit Intrusionen negativ, mit Vermeidung und Hyperarousal dagegen positiv. Die Strategie «Bestrafung» korreliert positiv mit Vermeidung und Hyperarousal, «Soziale Kontrolle» korreliert negativ mit Vermeidung. Zum zweiten Untersuchungszeitpunkt nach 3 Monaten korreliert lediglich noch die Strategie «Sorgen» negativ mit dem Auftreten von Intrusionen und Vermeidung. Die anderen vier Gedankenkontrollstrategien korrelieren nur in minimalem Ausmaß und nicht signifikant und weisen damit keinen signifikanten linearen Zusammenhang mit den PTB-Symptomclustern auf. Auch mit der dreistufigen Diagnose zum zweiten Untersuchungszeitpunkt (kein PTB, subsyndromales PTB, PTB) finden sich keine signifikanten Korrelationen.

\section{Gemeinsame Regressionsanalyse}

Mittels einer multiplen linearen Regressionsanalyse wurde getestet, welche Kombination von Variablen die Ausprägung der drei Symptomcluster des IES-R (Intrusionen, Vermeidung und Hyperarousal) 3 Monate nach dem Unfall am besten vorhersagt. Die erste Analyse testete die Prädiktion der PTB-Symptomatik durch die Kontrollvariablen Alter und Geschlecht und die traditionell untersuchte Variable der Verletzungsschwere. In der zweiten Analyse wurden die fünf TCQ-Subskalen mit einbezogen, um deren Beitrag für die Vorhersage der Symptomatik zu prüfen. In einem dritten Analyseschritt wurden zusätzlich die Interaktionen der IES-R-Intrusionsskala mit den TCQ-Subskalen zum ersten Untersuchungszeitpunkt berücksichtigt. Dieser Schritt diente der Prüfung, ob das gleichzeitige Vorliegen von Intrusionen und der Kontrollstrategie «Ablenkung» am ersten Zeitpunkt zur Aufklärung der PTB-Symptomatik am zweiten Zeitpunkt beiträgt.

\section{Vorhersage von Intrusionssymptomen}

Die Variablen Alter, Geschlecht und Verletzungsschwere erbringen in einem ersten Schritt der Regressionsanalyse keinen signifikanten Beitrag zur Varianzaufklärung der IES-RIntrusionsskala zum zweiten Untersuchungszeitpunkt. Auch die im zweiten Schritt hinzugenommenen fünf Subskalen des TCQ erbringen keinen signifikanten Zuwachs zur Varianzaufklärung. Erst durch die weitere Hinzunahme der Interaktionen zwischen dem Vorliegen von Intrusionen und Gedankenkontrollstrategien zum ersten Zeitpunkt wird in einem dritten Schritt eine signifikante Veränderung des $\mathrm{R}^{2}$ für das IES-RSymptomcluster Intrusionen zum zweiten Zeitpunkt erreicht ( $\mathrm{R}^{2}$-Change $\left.=0,20, \mathrm{~F}=2,64\right)$. Dabei weisen die Interaktionen von Intrusionen und den Kontrollstrategien «Ablenkung» und «Selbstbestrafung» positive $\beta$-Gewichte auf, die Strategie 


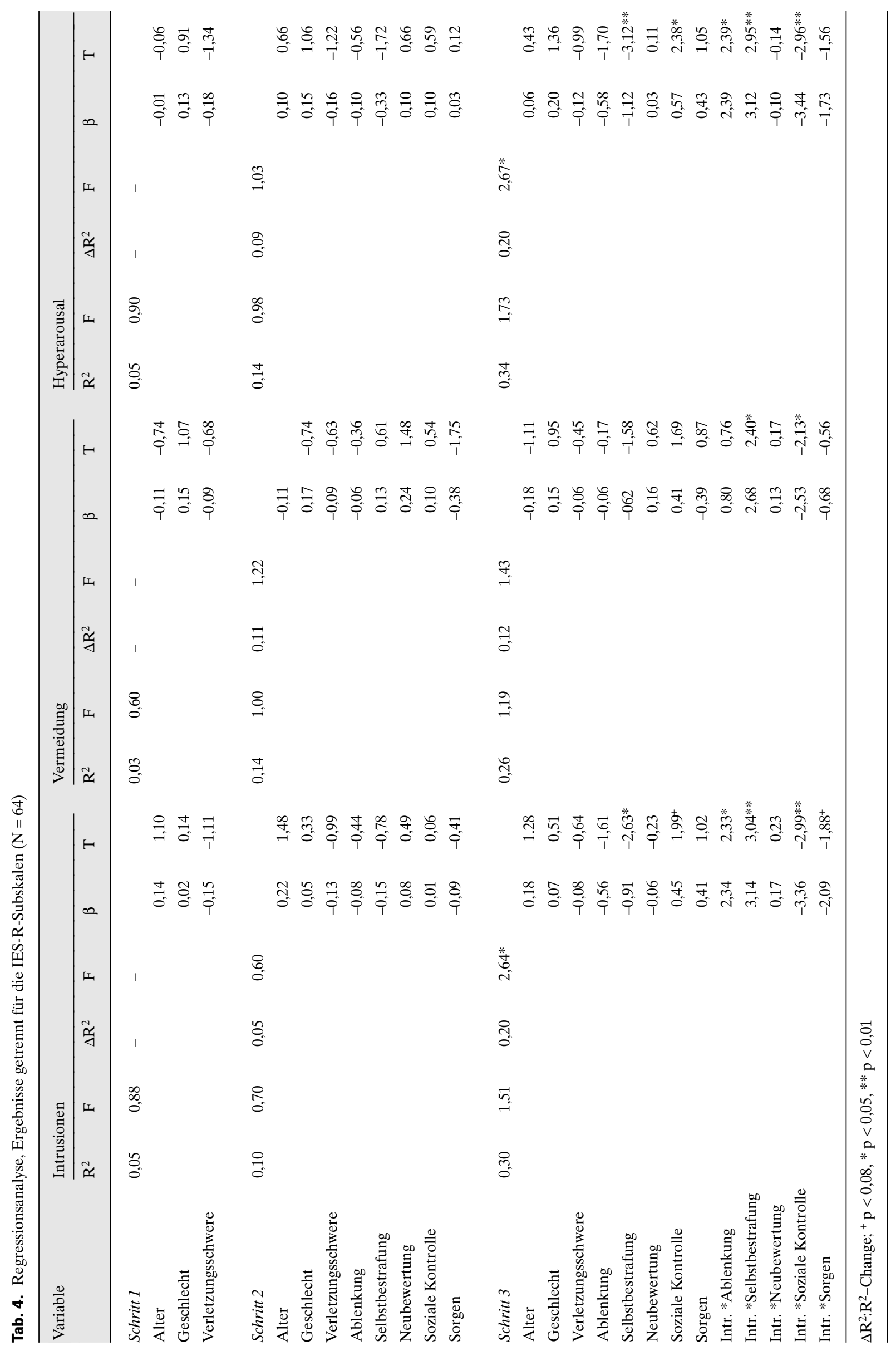


Selbstbestrafung sowie die Interaktion von Intrusionen und der Strategie «Soziale Kontrolle» weisen dagegen ein negatives $\beta$-Gewicht auf (Tab. 4).

\section{Vorhersage von Vermeidungssymptomen}

Hier konnte weder durch Hinzunahme der TCQ-Subskalen im zweiten Schritt noch durch die Einbeziehung der Interaktionen zwischen der IES-R-Intrusionsskala und den TCQSubskalen eine signifikante Zunahme des $\mathrm{R}^{2}$ erreicht werden. Dessen ungeachtet wiesen im dritten Schritt die Interaktion zwischen Intrusionen und Selbstbestrafung ein positives, die Interaktion zwischen Intrusionen und Sozialer Kontrolle ein negatives signifikantes $\beta$-Gewicht auf.

\section{Vorhersage von Hyperarousalsymptomen}

Die ersten beiden Regressionsschritte ergaben keine signifikanten Werte. Im dritten Regressionsschritt erbrachte die Hinzunahme der Interaktionsvariablen eine signifikante Änderung des $R^{2}(0,20 ; F=2,67)$. Dabei erreichten die Variable «Soziale Kontrolle» sowie die Interaktionsvariablen «Intrusion*Ablenkung»und «Intrusion*Selbstbestrafung» signifikante positive $\beta$-Gewichte. Die Variablen Selbstbestrafung sowie die Interaktionsvariable «Intrusion*Soziale Kontrolle» wiesen dagegen negative signifikante $\beta$-Gewichte auf (Tab. 4).

\section{Diskussion}

Die ermittelte Häufigkeit posttraumatischer Belastungssymptomatik im praxisrelevanten Setting einer unfallchirurgischen Klinik liegt in dem Bereich, den die Ergebnisse anderer Studien erwarten ließen. Es zeigte sich, daß etwa 10 Tage nach einem Verkehrsunfall 14\% der untersuchten Unfallpatienten an einer klinisch relevanten Belastungssymptomatik (PTB-Äquivalent) leidet. Drei Monate später hat die Prävalenzrate deutlich zugenommen und liegt bei 39\%. Betrachtet man die Symptomatik detailliert, so berichten zwei Drittel der Patienten zum ersten Untersuchungszeitpunkt von Intrusionen, die Hälfte von Vermeidung und ein Drittel von Hyperarousalsymptomen. 3 Monate später ist die Häufigkeit von Intrusionen und Hyperarousal weiter angestiegen, die Häufigkeit von Vermeidungssymptomen dagegen ist etwa gleich geblieben.

Interessant sind auch die Verläufe zwischen den beiden Untersuchungszeitpunkten. So verschlechtert sich ausgehend vom ersten Zeitpunkt bei 35\% der Zustand, 55\% bleiben mehr oder weniger unverändert, $10 \%$ remittieren. Diese Veränderungen beziehen sich auf die zwei in dieser Studie zu vergebenden diagnostischen Kategorien, d.h., ob eine PTB vorliegt oder nicht.
Frauen klagen zum ersten Untersuchungszeitpunkt häufiger über Intrusionen und Vermeidung als Männer und sie leiden nach 3 Monaten erwartungsgemäß häufiger unter PTB. Dies deckt sich mit bisherigen Ergebnissen aus der Psychotraumatologie sowie mit den Befunden zu «benachbarten» Störungskategorien wie Angsterkrankungen und Depression. Darüber hinaus sind ältere Unfallopfer häufiger von einer PTB-Symptomatik betroffen als jüngere zum zweiten Untersuchungszeitpunkt nach 3 Monaten.

Die Verletzungsschwere und die subjektiv eingeschätzte Schwere des Unfalls weist erwartungsgemäß keine substantielle Korrelation mit der PTB-Symptomatik auf. Dies deckt sich mit der Mehrzahl der Ergebnisse aus vergleichbaren Studien, vor allem solchen, die ebenfalls den ISS zur Bestimmung der Verletzungsschwere verwendeten. Es stützt neuere Befunde, nach denen weniger die objektivierbaren Aspekte des Traumas sondern vielmehr der kognitiv-emotionale bzw. behaviorale Umgang damit für die Entwicklung einer PTB von Bedeutung sind [Ehlers und Steil, 1995; Maercker, 1998]. Einschränkend ist jedoch anzumerken, daß die Verletzungsschwere nur eine unzureichende Operationalisierung der Unfallschwere darstellt, da sie lediglich die physischen Folgen beschreibt. Um aber die Unfallschwere angemessen zu operationalisieren, müßten weitaus mehr Aspekte des Verkehrsunfalls erfaßt und entsprechend ihres Beitrages gewichtet werden. Dies wäre eine Aufgabe für weitere Forschung.

Alle fünf Subskalen des TCQ korrelieren mit der posttraumatischen Belastungssymptomatik nach einer Woche (Tab. 3). Am stärksten sind hier die Zusammenhänge bei den Skalen Ablenkung, Sorgen und Bestrafung. Eher geringe Zusammenhänge finden sich bei den Skalen Neubewertung und soziale Kontrolle. Da es sich hierbei lediglich um korrelative Zusammenhänge handelt, ist nicht klar, ob beispielsweise aufgrund von häufig auftretenden Intrusionen Ablenkungsstrategien eingesetzt werden oder ob aufgrund von Ablenkungsstrategien die Intrusionen gehäuft auftreten. Beides wäre plausibel. Hier könnten die Korrelationen zwischen Gedankenkontrollstrategien $\mathrm{zu}_{1}$ und PTB-Symptomatik zu t $\mathrm{t}_{2}$ Aufschluß geben. Es zeigt sich, daß lediglich die Strategie «Sorgen» signifikant negativ korreliert und zwar mit Intrusionen und Vermeidung. Dies könnte bedeuten, daß je stärker Menschen sich kurz nach dem Unfall in sorgenvoller, grüblerischer Weise mit den Gedanken daran beschäftigen, desto seltener mittelfristig Intrusionen auftreten. Dabei haben diese Menschen das Gefühl, sich in Form von Sorgen mit dem Trauma auseinanderzusetzen und damit das Trauma gerade nicht zu vermeiden. So läßt sich eventuell die negative Korrelation mit den Vermeidungssymptomen erklären, die auf den ersten Blick keinen Sinn zu machen scheint.

Auf der Suche nach dem prädiktiven Wert der erhobenen Gedankenkontrollstrategien wurde eine multiple hierarchische Regressionsanalyse in drei Schritten durchgeführt. Dabei konnte erst nach Hinzuziehung der Interaktionen zwischen Intrusionen und Gedankenkontrollstrategien $\mathrm{zu}_{\mathrm{t}}$ ein signifi- 
kanter Zuwachs des $\mathrm{R}^{2}$ für die Prädiktion von Intrusionen und Hyperarousal erreicht werden (Change- $R^{2}$ jeweils 0,20). Mit den in die Regressionsanalyse einbezogenen Variablen konnten 30\% der Varianz der Intrusionsskala zu $\mathrm{t}_{2}$ sowie $34 \%$ der Hyperarousalskala zu $\mathrm{t}_{2}$ aufgeklärt werden. Den stärksten Beitrag zur erreichten Varianzaufklärung dieser beiden Symptomcluster bzw. die höchsten signifikanten $\beta$-Gewichte erbrachten die Interaktionsvariablen «Intrusion*Ablenkung», «Intrusion*Selbstbestrafung» (positive $\beta$-Gewichte) und «Intrusion*Soziale Kontrolle» (negative $\beta$-Gewichte). Das heißt: Bei Vorliegen von Intrusionen und gleichzeitigem Einsatz der Gedankenkontrollstrategien «Ablenkung» oder «Selbstbestrafung» kurz nach dem Unfall ist mit einer erhöhten Auftretenswahrscheinlichkeit von Intrusionen und Hyperarousal 3 Monate später zu rechnen. Das gemeinsame Vorliegen von Intrusionen und der Gedankenkontrollstrategie «Soziale Kontrolle» $\mathrm{zu} \mathrm{t}_{1}$ senkt dagegen eher die Auftretenswahrscheinlichkeit von Intrusionen und Hyperarousal zu $t_{2}$.

Was bedeuten diese Interaktionseffekte? Sie sind Hinweise darauf, daß weder das Auftreten von Intrusionen allein noch der Einsatz von Gedankenkontrollstrategien, die im Allgemeinen eher als dysfunktional betrachtet werden, die Aufrechterhaltung bzw. Neuentwicklung einer PTB-Symptomatik vorhersagen. Erst bei gleichzeitigem Vorliegen beider Faktoren erhöht sich die Wahrscheinlichkeit einer PTB-Symptomatik 3 Monate später. Im übrigen deckt es sich mit Befunden von Reynolds und Brewin [1998], die bei PTB-Patienten die Strategie «Ablenkung» beim Umgang mit Intrusionen signifikant häufiger fanden als in einer gesunden Kontrollgruppe. Interessant ist weiterhin, daß bestimmte Gedankenkontrollstrategien selektive Effekte zu haben scheinen. Versuchen die Patienten, ihre Intrusionen mittels Ablenkung oder Selbstbestrafung zu bewältigen, werden sie eher aufrechterhalten.
Tauschen sich die Patienten dagegen mit anderen über die Intrusionen aus (Strategie «Soziale Kontrolle»), so scheint dies eher zu einem Abklingen der Intrusionen und der Hyperarousalsymptome beizutragen. Hier scheinen sich die bekannten Effekte vor allem kognitiver Vermeidung widerzuspiegeln, die unter anderem aus dem Bereich der Angst- und Zwangsstörungen bekannt sind. Entgegen den Erwartungen zeigte sich kein positiver, d.h. symptomreduzierender Effekt der Gedankenkontrollstrategie «Neubewertung». Gerade diese Art des Umgangs mit intrusiven Erinnerungen an ein traumatisches Ereignis wird gemeinhin als wichtiger Faktor für die erfolgreiche Bewältigung eines Traumas angesehen. Auch die Gedankenkontrollstrategie «Sorgen» wies nicht den erwarteten (aufrechterhaltenden) Effekt auf. Woran könnte das liegen? Zunächst muß berücksichtigt werden, daß diese differentiellen Befunde explorativ sind und noch weiterer Forschung bedürfen. Darüber hinaus könnten folgende Gründe in Frage kommen: mangelnde divergente Validität der Subskala «Sorgen» oder auch unzureichende konvergente Validität bzw. Konstruktvalidität des TCQ. So zitiert Fehm [1997] eine Untersuchung von Freeston et al. [1995], die nahelegt, daß ein wesentlich breiteres Repertoire von Strategien zum Umgang mit unerwünschten Gedanken zur Verfügung steht, als im TCQ erfaßt. Dazu kommt, daß je nach Erfolg der eingesetzten Strategie selbstverständlich auch mehrere Strategien kombiniert oder hintereinander eingesetzt werden können. Dies legt nahe, daß die Erfassung von Gedankenkontrollstrategien mittels eines Fragebogens mit fünf Subskalen unter Umständen zu stark verallgemeinert und daß dadurch vorhandene Unterschiede verwischt werden könnten. Hier scheint ein wesentliches Potential zur Erhöhung der Aussagekraft von Studien zum Thema Umgang mit Intrusionen zu stecken.

\section{Literatur}

AMA Commitee on Medical Aspects of Automotive Safety: Rating the severity of tissue damage. I. The Abbreviated Scale. J Am Med Assoc 1971;215:277280.

American Psychiatric Association: Diagnostic and Statistical Manual of Mental Disorders. (4th ed). Washington, Am Psych Assoc, 1994.

Baker SP, O'Neill B, Haddon W, Long WB: The Injury Severity Score: A method for describing patients with multiple injuries and evaluating emergency care. J Trauma 1974;14:187-196.

Blanchard EB, Hickling EJ, Mitnick N, Taylor AE, Loos WR Buckley TC: The impact of severity of physical injury and perception of life threat in the development of posttraumatic stress disorder in motor vehicle accident victims. Behav Res Ther 1995;33:529534.

Blanchard EB, Hickling EJ, Taylor AE, Loos WR, Forneris CA Jaccard J: Who develops PTSD from motor vehicle accidents?. Behav Res Ther 1996;34:1-10. Bryant B, Harvey AG: Avoidant coping style and posttraumatic stress following motor vehicle accidents. Behav Res Therapy 1995;33:326-336.
Clark DA, Purdon C: New perspectives for a cognitive theory of obsessions. Aust Psychologist 1993;28: 161-167.

Ehlers A, Steil R, Winter H, Foa EB: Deutsche Übersetzung der Posttrauamtic Stress Diagnostic Scale (PDS). University of Oxford. Unveröffentlichtes Manuskript, 1996.

Ehlers A, Mayou RA, Bryant B: Psychological predictors of chronic posttraumatic stress disorder after motor vehicle accidents. J Abnorm Psychol 1998;107:508519 .

Ehlers A, Steil R: Maintenance of intrusive memories in posttraumatic stress disorder: A cognitive approach. Behav Cogn Psychother 1995;23:217-250.

Fehm L: Diagnostik unerwünschter Gedanken. Unveröffentlichtes Manuskript. Dresden, Christoph-Dornier-Stiftung für Klinische Psychologie, 1997.

Feinsten A, Dolan R: Predictors of post-traumatic stress disorder following physical trauma: An examination of the stressor criterion. Psychol Med 1991;21: 85-91.

Foa EB: PDS (Posttraumatic Stress Diagnostic Scale) Manual. Minneapolis, National Computer Systems, 1995.
Foa EB, Cashman L, Jaycox L, Perry K: The validation of a self-report measure of posttraumatic stress disorder: The Posttraumatic Stress Diagnostic Scale. Psychol Assess 1997;9:445-451.

Freeston MH, Ladoucer R, Provencher M, Blais F Strategies used with intrusive thoughts: Context, appraisal, mood and efficacy. J Anxiety Disord 1995;9: 201-215.

Frommberger U, Schlickewei W, Nyberg E, Stieglitz R-D, Kuner E, Berger M: Die psychischen Folgen von Verkehrsunfällen. Ergebnisse einer prospektiven Studie. Unfallchirurgie 1998;3:122-128.

Hickling EJ, Blanchard EB: Posttraumatic stress disorder and motor vehicle accidents. J Anxiety Disorders 1992;6:285-291.

Kessler RC, Sonnega A, Bromet E, Nelson CB: Posttraumatic stress disorder in national comorbidity survey. Arch Gen Psychiatry, 1995;52:1048-1060.

Kilpatrik DG, Saunders BE, Amick-McMullen A, Best CL, Veronen LJ, Resnick, HS: Victim and crime factors associated with the development of crime-related posttraumatic stress disorder. Behav Ther 1989;20: 199-214. 
Koren D, Arnon I, Klein E: Acute stress response and posttraumatic stress disorder in traffic accident victims: A one-year prospective follow-up study. Am J Psychiatry 1999;156:367-373.

Maercker A: Erscheinungsbild, Erklärungsansätze und Therapieforschung; in Maercker A (Hrsg): Therapie der posttraumatischen Belastungsstörungen. Berlin, Springer, 1997.

Maercker A: Posttraumatische Belastungsstörungen. Psychologie der Extrembelastungsfolgen bei Opfern politischer Gewalt. Lengerich, Pabst, 1998.

Maercker A, Schützwohl M: Erfassung von psychischen Belastungsfolgen: Die Impact of Event Scale revidierte Version (IES-R). Diagnostica 1998;44:130 141.

Malt UF: The longterm psychiatric consequences of accidential injuries. A longitudinal study of 107 adults. Br J Psychiatry 1998:153:810-818.

Malt UF, Hoivik B, Blikra G: Psychosocial consequences of road traffic accidents. Eur Psychiatry 1993;8: 227-228.

March JS: What constitutes a stressor? The „criterion A“ issue; in Davidson JRT, Foa EB (eds): Posttraumatic Stress Disorder: DSM-IV and beyond. Washington, American Psychiatric Press, 1993, pp 37-54.

Mayou R, Bryant B, Duthie R: Psychiatric consequences of road traffic accidents. Br Med J 1993;307:647651.

Norris FH: Epidemiology of trauma: Frequency and impact of different potentially traumatic events on different demographic groups. J Consult Clin Psychol 1992;60:409-418.

Nyberg E, Frommberger U, Stieglitz R-D, Berger M Langzeitverlauf Posttraumatischer Belastungsstörungen nach Verkehrsunfällen. Ergebnisse einer prospektiven Studie mit drei Meßzeitpunkten. Vortrag auf dem 41. Kongreß der Deutschen Gesellschaft für Psychologie, 27.09.-01.10.1998, Dresden, 1998.

Reynolds M, Brewin CR: Intrusive cognitions, coping strategies and emotional responses in depression, post-traumatic stress disorder and a non-clinical population. Behav Res Ther 1998;36:135-147.

Salkovskis PM, Campbell P: Thought suppression induces intrusion in naturally occurring negative intrusive thoughts. Behav Res Ther 1994:32:1-8.

Statistisches Bundesamt (Hrsg): Gesundheitsbericht für Deutschland. Stuttgart, Metzler-Poeschel, 1998, pp 155-157.

Steil R, Ehlers A, Clark DM: Kognitive Aspekte bei der Behandlung der posttraumatischen Belastungsstörung; in Maercker A (Hrsg): Therapie der posttraumatischen Belastungsstörungen. Berlin, Springer, 1997.

Wegner DM, Schneider DJ, Carter SR, White TL: Paradoxical effects of thought suppression. J Personality Social Psychol 1987;53:5-13.

Weiss DS, Marmar CR: The Impact of Event Scale revised; in Wilson JP, Keane TM (eds): Assessing Pychological Tauma and PTSD: A Handbook of Practitioners. New York, Guilford, 1997, pp 399-411.

Wells A, Davies ID: The Thought Control Questionnaire: A measure of individual differences in the control of unwanted thoughts. Behavior Research and Therapy, 1994;32:871-878.

Winton E, Clark DM, Ehlers A: Cognitive factors in persistant versus reco-vered posttraumatic stress disorder after physical or sexual assault: A pilot study. (submitted). 